RAD Conference Proceedings, vol. 2, pp. 243-248, 2017

www.rad-proceedings.org

\title{
ELECTROMAGNETIC FIELD THERAPY AND IMMUNE MECHANISMS WHICH ARE INVOLVED IN ANTI-INFLAMMATORY RESPONSE
}

\author{
Mihai Surcel ${ }^{1}$, Didi Surcel ${ }^{*}$, Sebastian Toader ${ }^{1}$, Mioara Butan² \\ ${ }^{1}$ University of Medicine and Pharmacy, Cluj Napoca, Romania \\ ${ }^{2}$ Blue Life Medical Center, Cluj Napoca, Romania
}

\begin{abstract}
The aim of this study was to evaluate the mechanism by which EMF therapy can interfere with the modified immune and oxidative reactions by the experimental approach on the lab animals. In vivo and vitro experiment was carried out on 60 Wistar rats that were divided in 4 groups as following: 1 . Control-group tested on the $10^{\text {th }}$ day; 2. LF-EMF-exposed group tested on the $10^{\text {th }}$ day; 3. Group that received intradermic Staphylococcus aureus culture $(S A)$ and was tested on the $10^{\text {th }}$ day; 4 . Group that received intradermic SA and was treated with $L F$ EMF and was tested after 10 days. The rats were exposed to $50 \mathrm{~Hz}, 1 \mathrm{mT}$. Splenic lymphocytes and alveolar macrophages for the cellular cultures were harvested. The following parameters were assessed: a) $3 H T d R$ incorporation test; b) Macrophage inhibition factor (MIF) assay; c) IL-1 assay; d) TNF-assay; e) Chemiluminescence assay; f) Phagocytosis assay. The $3 H T d R$ incorporation test, tests targeting the cytokine's activity and the phagocytosis test point out a partial reversibility of the values in the EMF-exposed groups that was treated with staphylococcus cultures. Our experiment reveals that the EMF interferes with the immune and oxidative systems, becomes chronic and plays a key role in the development of serious diseases. The EMF-therapy can be beneficial in reducing the inflammation by interfering with the inflammatory molecules and surface receptors.
\end{abstract}

Key words: ELF-EMF, immune reactions, cytokines, free radicals

DOI: $10.21175 /$ RadProc.2017.50

\section{INTRODUCTION}

There is presently an intense discussion whether the exposure to electromagnetic field (EMF) has consequences for human health. Various cellular components, processes and systems can be affected by the EMF exposure. Since it is unlikely that EMF can induce DNA damage directly, most studies have examined EMF effects on the cell membrane level, general and specific gene expression, and signal transduction pathways $[1,9,11]$. The immune system serves as the most important part of the general defense barrier against microbes and viruses, and EMFs can penetrate the entire body. EMFs disturb the immune functions through the stimulation or suppression reactions and by generation of free radicals $[2,9,11]$. Free radicals (FR) are intermediates in the natural processes like mitochondrial metabolism and are also a key feature of phagocytosis [13]. EMF might be a stimulus to induce an "activated state" of the cell such as phagocytosis, which then enhances the release of free radicals. In the recent years, the interest in the appliances that emit extremely low frequency electromagnetic field (ELF- EMF) has rapidly increased since the results that were very good in comparison with other treatments used for infections and for healing tissue [3, 14].

\section{MATERIALS AND METHODS}

The aim of this study was to evaluate the mechanism by which ELF- EMF therapy can interfere with the immune reactions and oxidative reactions by experimental approach in the lab animal study. Experimental study was performed on the 60 Male Wistar rats weighing 200-220 grams, grouped in 4 batches: 1.Controls; 2. ELF-EMF-exposed group (E), with animals' exposure to $5 \mathrm{oHz}, 1 \mathrm{mT}$ EMF, 6h / day, for 10 days; 3 . The group treated with the infectious agent that received, intradermally, Staphylococcus aureus (SA) culture; 4. Group that was exposed to ELF$\mathrm{EMF}$ and received SA $(\mathrm{E}+\mathrm{SA})$. After 10 days all animals were euthanized and sacrificed. splenic lymphocytes and alveolar macrophages were harvested and the cellular cultures were prepared for performing immunological and chemical tests. The splenic lymphocytes were obtained after density gradient centrifugation using the Ficoll - Na Metrizoat mixture after a prior trituration of the spleen. The sediment was taken up and washed, and after counting the cells in the Burker-Turck hemocytometer was adjusted to $1.10^{6}$ cells / ml. For 3 HTdR incorporation test in the cellular RPMI-1640 medium was added PHA to $\mathrm{T}$ cells stimulation and after $48 \mathrm{~h}$ was added in each culture

*didisurcel1@gmail.com 
$1 \mu \mathrm{Ci}$ of tritiated thymidine ( $3 \mathrm{HTdR})$. T cell cultures were filtered through Sartorius $\mathrm{nr} 11303$ wells and fixed with a $5 \%$ trichloroacetic acid solution. The welldried wells were placed in scintillation fluid and read on a radiation counter. The results were expressed in pulses per minute (cpm). For a macrophage inhibition migration (MIF -assay), AMs obtined by bronchoalveolar lavage (BAL), were performed by Soborg - Bendixen technique, method (indirect test) in which the supernatant of lymphocyte cultures was used in $1 / 4$ dilution and the indicator cells were the guinea pig peritoneal macrophages. After 18 hours of migration at $37^{\circ} \mathrm{C}$ the macrophage migration areas were measured by means of an optical microscope adapted to the optical microscope. The results were expressed in micrometres and in migration indices. Alveolar macrophages were tested for cytokine release (IL-1 assay, TNF -assay), free oxygenated radicals (ROS) , revealed by the chemiluminescence assay and phagocytosis. The IL-1 assay was performed by the Mizel method. Mice tymocites, 1x106 cells / ml, were grown in RPMI-1640 medium in the presence of $1 \mu \mathrm{g} /$ $\mathrm{ml}$ PHA and $0.2 \mathrm{ml}$ of the supernatant of AMs stimulated cultures LPS, diluted 1/20. Twenty-four hours after incubation $0.5 \mu \mathrm{l}$ of $3 \mathrm{Hdd} \mathrm{R}$ was added and after another 18 hours the cultures were sacrificed. The results were expressed in pulses per minute (cpm)

TNF -assay was performed by the Flick and Gifford method, slightly modified by us. Suspension of the L929 tumor cell in a concentration of $2{\mathrm{X} 10^{4}}^{4}$ cells / $\mathrm{ml}$ in the presence of $24 \mu \mathrm{g} / \mathrm{ml}$ actinomycin D and of the supernatant of the AMs + LPS cultures in 1/20 dilution were cultured for 48 hours in RPMI-1640 medium containing $1 \mu \mathrm{Ci} / \mathrm{ml}$ of $3 \mathrm{HTdR}$ with specific activity of $19 \mathrm{Ci}$. The cultures were sacrificed after 18 hours of incubation and prepared for reading at the radiation counter. The results were processed with the T Student test.For chemiluminiscence assay, AMs were stimulated with zymosan. It was used a Beckman liquid scintillation cunter. For phagocytosis, alveolar macrophage cultured in RPMI-1640, were adjusted to the concentration of 2.105. After the adhesion of AMs from the cell suspension, the EA system (SRBC sensitized with $0.25 \%$ antiserum) was added in equal number , 2X105 of Staphylococcus aureus (S A). After incubation at 30 'and 180', the smears were wash, dry and color with Giemsa. The results were expressed in the percentage of AMs that contain SA and number of AMs that are contained in AMs after 180'.

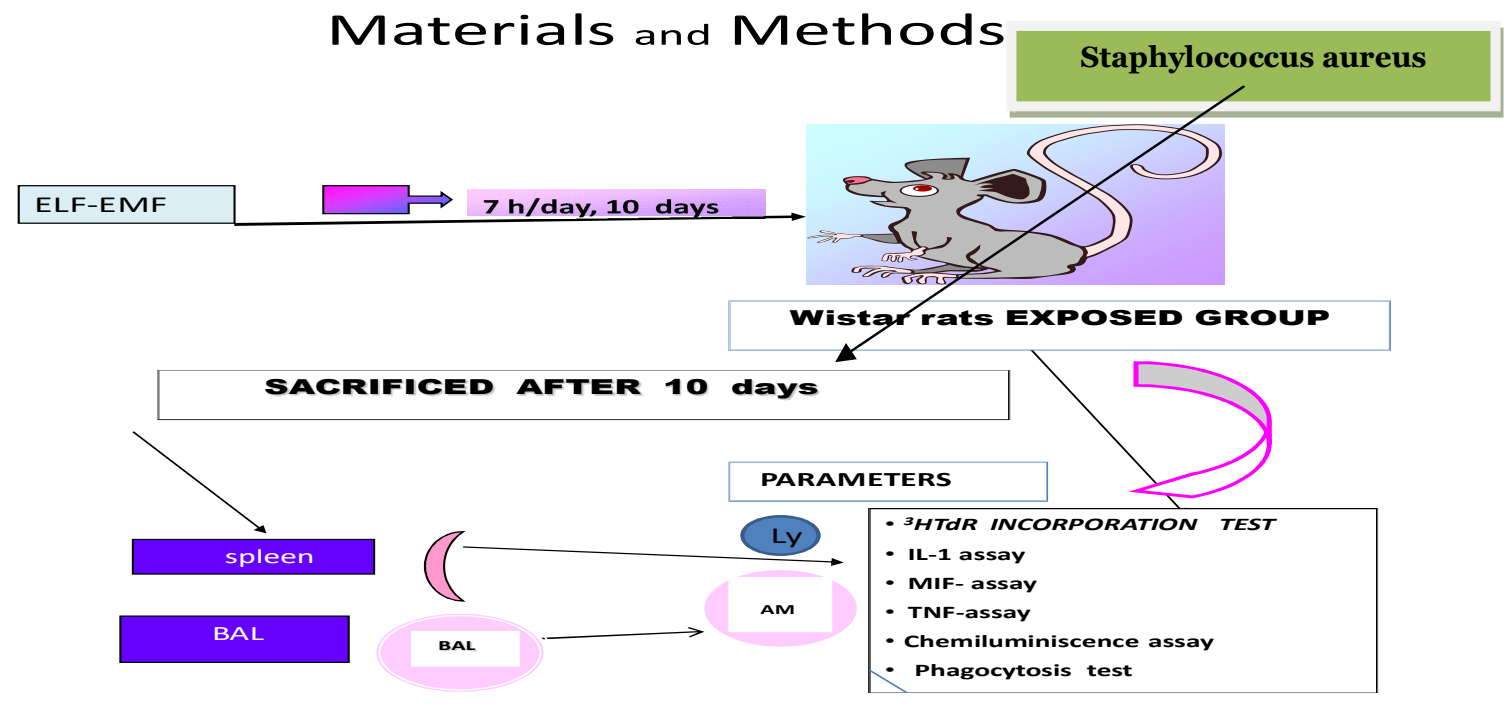

Figure 1. Framework

Statistical processing - the results were processed using the Student's t-test, arithmetic mean (X), standard deviation (d), percentage changes compared to the control and test probability (p).

\section{RESULTS AND DISCUSSION}

The effects of the ELF-EMF exposure of the Wistar rats, according to the experimental model presented, point out the following changes in the investigated immunological and inflammatory tests.
3 HTdR incorporation test (Fig. 2) in the EMF-ELFexposed group (E) reveals values of the incorporation of $3 \mathrm{HTdaR}$ in the splenic lymphocytes very close to those of the control group (C), without statistically significant differences (no SSD). In the group with Staphylococcus aureus (SA), the values of the incorporation of $3 \mathrm{HtdR}$ are decreased, with $\mathrm{SSD}$, in comparison with $\mathrm{C}$. In the $\mathrm{E}+\mathrm{SA}$ group, the values of the incorporation of the $3 \mathrm{HtdR}$ are intermediate, suggesting a protective role of the EMF-ELF on the lymphocyte proliferation, when there is an infectious and inflammatory process. 


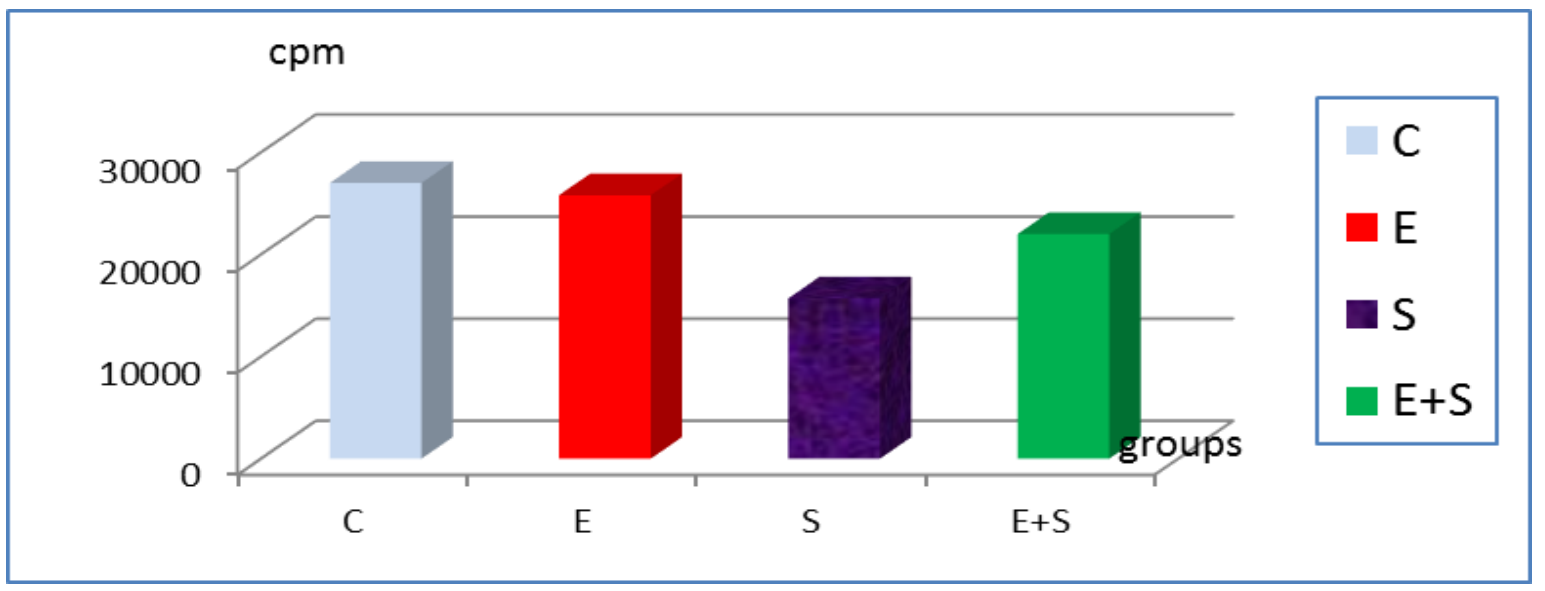

Figure 2. 3HTdR incorporation test in splenic lymphocytes after 10 days in the exposed rats.

Effect of the ELF-EMF exposure on the $3 \mathrm{HTdR}$ incorporation in the rats treated with SA

IL-1 assay (Fig. 3) points out the effect of the exposure to ELF-EMF on the IL-1 release by AMs obtained by BAL, after 10 days of the exposure of the rats. In the E group, the values of the IL-1 are similar to those of the control group, without SSD. In the SA group, the values of the IL-1 are increased, with SSD, in comparison with the control group. In the E+ SA group, the values of the IL-1 are intermediate, suggesting the involvement of the EMF in the IL-1 production, this cytokine being a constant presence of the inflammatory process.

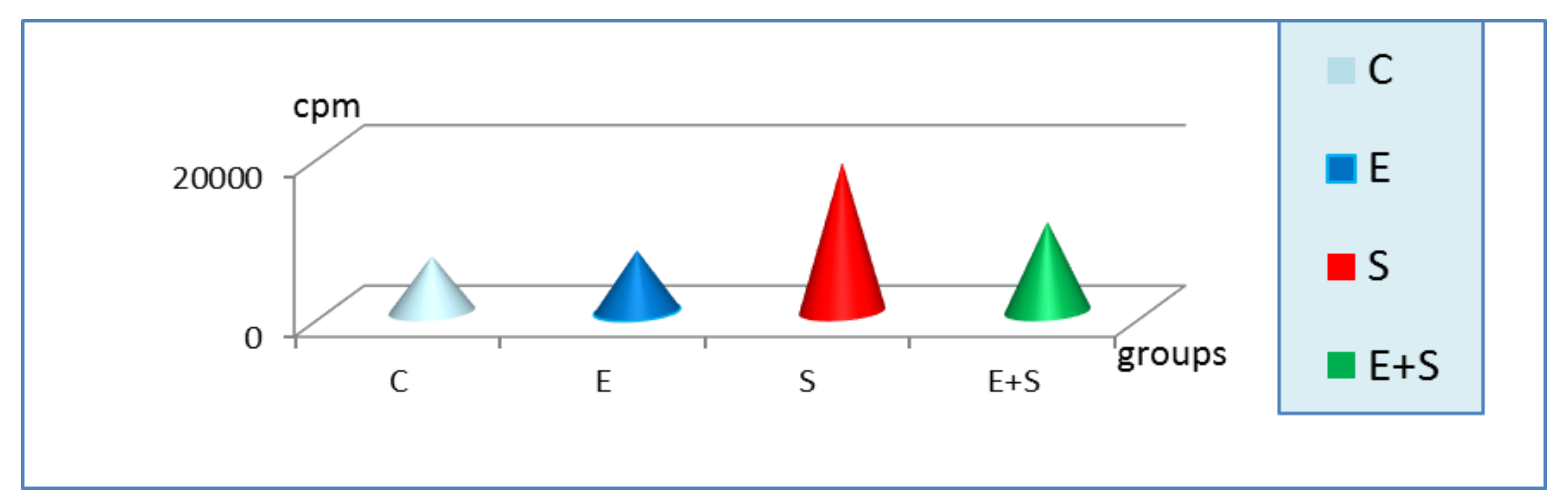

Figure 3. IL-1 assay. Effect of the exposure to ELF-EMF on the IL-1 release by AMs obtained by BAL, after 10 days of the exposure of the rats

MIF-assay (Fig. 4) reveals the effect of the exposure to ELF-EMF on the macrophage inhibition factor (MIF) released by the splenic lymphocytes, collected after 10 days of the rats' exposure and cultured in the presence of the alveolar macrophages. In the E group, the values of the MIF were similar to those of the controls, but in the SA group, the values of the MIF were decreased, with SSD, in comparison with control, suggesting an inflammatory process. The partial reversibility of the MIF values in the $\mathrm{E}+\mathrm{SA}$ group, suggests an interference with this cytokine, generated by the inflammatory process. 


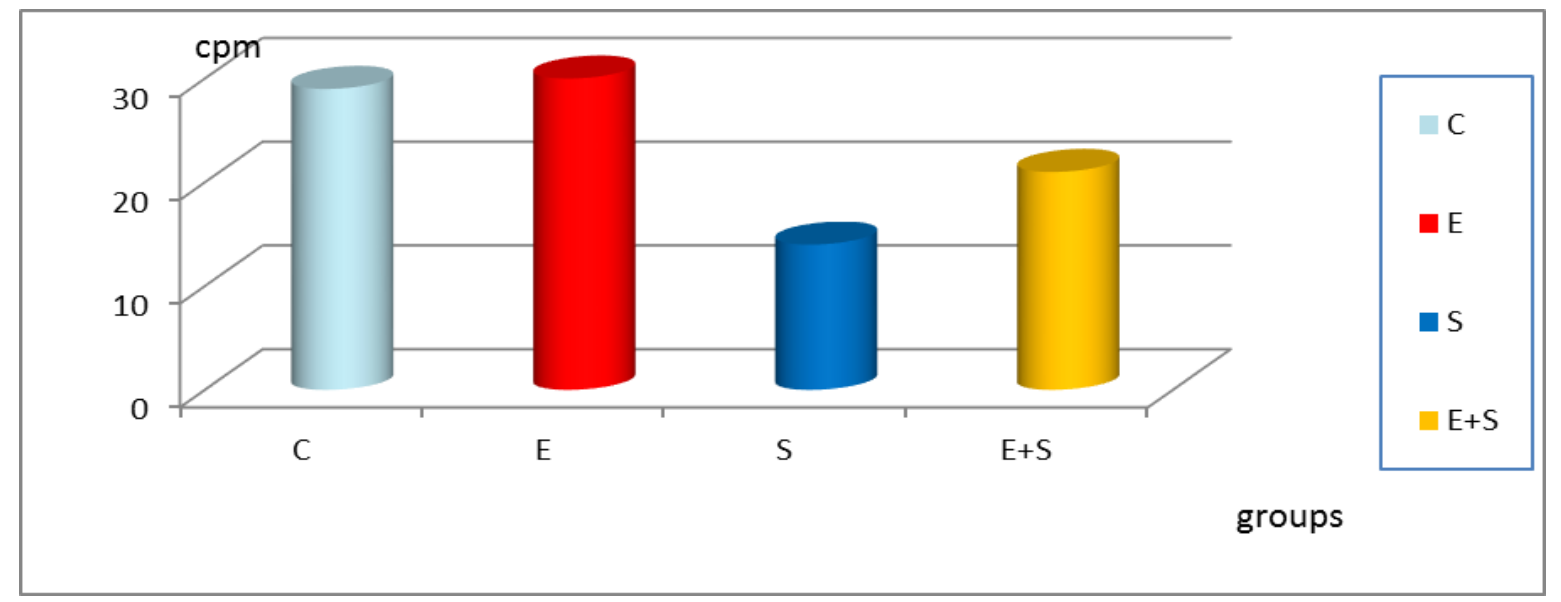

Figure 4. Effect of the exposure to ELF-EMF on the MIF released by splenic Lys, cultured in the presence of macrophages, after 10 days of the rats' exposure

TNF-assay reveals the effect that simultaneous exposure to the SA and ELF-EMF has on the rats concerning the TNF release. AMs were obtained by BAL, 10 days after the rats' exposure (Fig. 5).

In the E group, the values of the TNF are similar to those in the control group, without SSD. In the SA group, the values of the TNF are increased, with SSD, in comparison with controls. In the $\mathrm{E}+\mathrm{SA}$ group, the values of the TNF are more increased than in the SA group, but without SSD. In comparison with controls, the TNF values in this group are increased, with SSD. These results reveal an increased involvement of this cytokine in the inflammatory reactions, induced by a pathogen agent that also increases the level of TNF, representing the effort of the body to control pathogeninduced tissue injury.

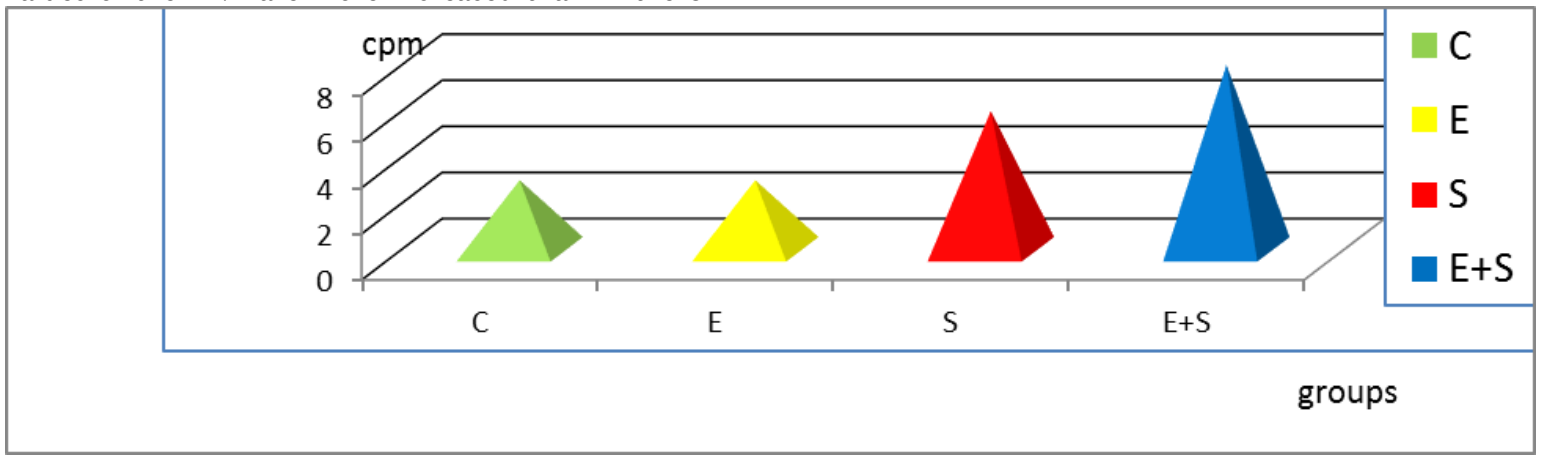

Figure 5. TNF-assay. Effect of the exposure to ELF-EMF on the TNF release by rats' AMs, obtained by BAL, 10 days after exposure of the rats

Luminol-dependent chemiluminescence assay points out the effect of the exposure of the rats to the ELF-EMF, leading to the ROS release from the alveolar macrophages (AMs), obtained by BAL, 10 days after the exposure (Fig. 6). In the E exposed group, the values of the ROS are similar to those in the control group, without SSD. In the SA group, the values of the radical oxygen species (ROS) are increased, with SSD, in comparison with the controls. In $\mathrm{E}+\mathrm{SA}$ group, the values of the ROS are intermediate, with SSD, both in comparison with controls and with SA group. These results suggest an improvement of the EMF-ELF in the oxidative reactions, developed in the inflammatory process. Decrease of the ROS in the E + SA group, evidenced by the partial restoration of the chemiluminescence values, is the proof of the decrease of the inflammatory process. 


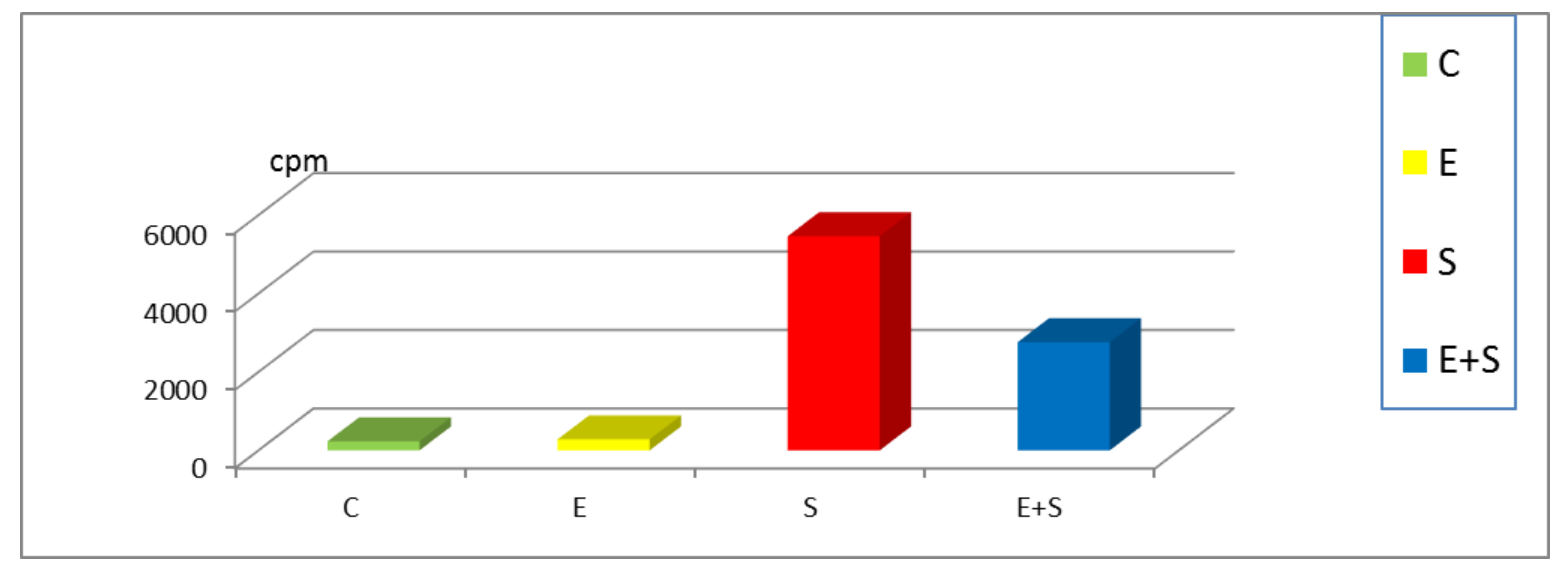

Figure 6. Luminol-dependent chemiluminescence assay points out the effect of the exposure of the rats to the ELF-EMF, aiming to provide the evidence of the ROS released from the alveolar macrophages (AMs), obtained by BAL, 10 days after the exposure

Phagocytosis assay points out the percentage of macrophages (AMs) that are in the phagocytic state and the effect of the ELF-EMF exposure on the phagocytic process of the AMs (Fig. 7).

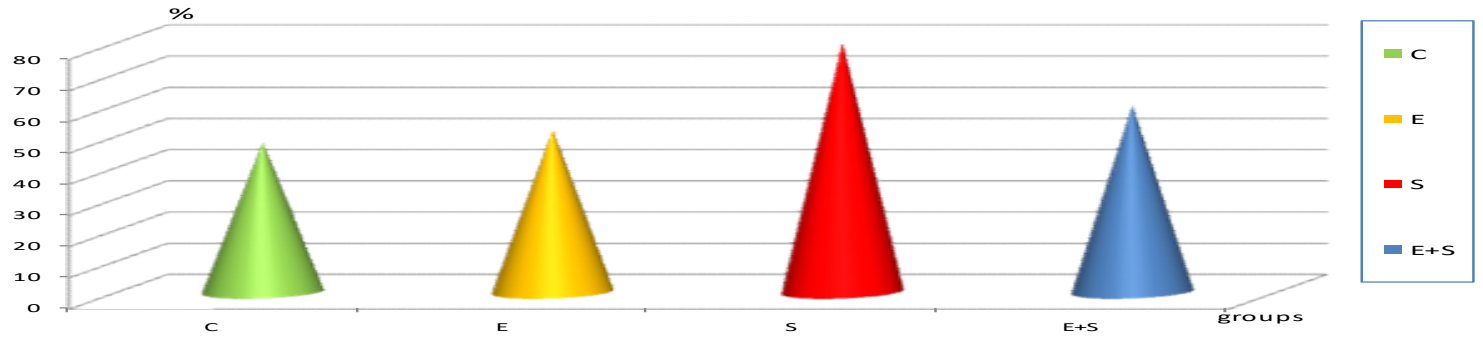

Figure 7. Phagocytosis assay. The percentage of AM that are in phagocytic state. Effect of the ELF-EMF exposure on the phagocytic macrophages

In the EMF-ELF-exposed group, AMs that are in the state of phagocytosis are similar to those in the control group, without SSD. In the SA group, the percentage of the phagocytic AMs is very increased, with SSD, in comparison with the control group. In the $\mathrm{E}+\mathrm{SA}$ group, the percentage of the phagocytic AMs is intermediate, suggesting the involvement of the EMFELF in the phagocytosis process by which high levels of ROL are released for the removal of the pathogenic agent. Wound healing is a highly coordinated and complex process involving the proliferation and migration of the various cell types, especially inflammatory cells, chemical mediators and the surrounding extracellular matrix, resulting in a tightly orchestrated re-establishment of tissue integrity. ELFEMF interact with the immune response and particularly with the activity of the monocyte -derived macrophages that fight against the pathogens by releasing ROS and cytokines. Inflammatory cytokines are intermediates in the inflammatory process, while free radicals are intermediates in the natural processes like mitochondrial metabolism and a key feature of phagocytosis $[1,3,9]$. Low-frequency electric fields influence all systems characterized by charged particles as the human body. In fact, tiny electrical currents exist in the human body, due to the chemical reactions that occur as a part of the normal bodily functions, even in the absence of the external electric fields. For example, nerves transmit signals through the electrical impulses. The amplification of the signal to a biological response in past years has been thought to be driven solely by the chemical messengers and receptors, but now, there is evidence that the molecular mechanisms may not be the only factors involved in the biological communication. Chemical messengers are perhaps the particle components modulated by the governing energy fields which underlie the signal transduction $[4,5]$. Our results highlight the beneficial effects of the ELF-EMF on the immune reactions by the stimulation of the $\mathrm{T}$ lymphocytes proliferation and by the regulation of the cytokines and free radicals, biological processes that are necessary in the healing of wounds.

\section{Conclusions}

Our experiment reveals that the EMF interferes with the immune and oxidative systems. ELF-EMF has no harmful effect on the healthy tissue. ELF-EMF interferes with the inflammatory and immune reactions that accompany inflammatory processes, induced by the infectious, physical or chemical agents. ELF-EMF-therapy can be beneficial in reducing the inflammation by interfering with the inflammatory molecules and surface receptors. 


\section{REFERENCES}

1. M. Simkó, M. Mattsson, "Extremely low frequency electromagnetic fields as effectors of cellular responses in vitro: possible immune cell activation," J. Cell Biochem., vol. 93, no. 1, pp 83 - 92, Sep. 2004.

DOI: $10.1002 /$ jcb.20198

PMid: 15352165

2. D. B. Lyle, R. D. Ayotte, A. R. Sheppard, W. R. Adey, "Suppression of T-lymphocyte cytotoxicity following exposure to $60-\mathrm{Hz}$ sinusoidal electric fields," Bioelectromagnetics, vol. 9, no. 3, pp. 303-313, 1988. DOI: $10.1002 /$ bem.2250090311 PMid: 3263132

3. C. D’Angelo, E. Costantini, M. A. Kamal, M. Reale, "Experimental model for elf-emf exposure: concern for human health," Saudi J. Biol. Sci., vol. 22, no. 1, pp. 75 - 84, Jan. 2015.

DOI: $10.1016 /$ j.sjbs.2014.07.006

PMid: 25561888

PMCid: PMC4281612

4. M. L. Guzman, S. J. Neering et al., "Nuclear factor- $\kappa \mathrm{B}$ is constitutively activated in primitive human acute myelogenous leukemia cells," Blood, vol. 98, no. 8, pp. $2301-2307$, Oct. 2001.

DOI: 10.1182/blood.V98.8.2301

PMid: 11588023

5. A. Maziarz, B. Kocan, M. Bester, S. Budzik, "How electromagnetic fields can influence adult stem cells: positive and negative impacts," Stem Cell Res. Ther., vol. 7, no. 1, p. 54, Apr. 2016.

DOI: $10.1186 / \mathrm{s} 13287-016-0312-5$

PMid: 27086866

PMCid: PMC4834823

6. S. Gangi, O. Johansson, "A theoretical model based upon mast cells and histamine to explain the recently proclaimed sensitivity to electric and/or magnetic fields in humans," Medical Hypotheses, vol. 54, no. 4, pp. 663 - 671, Apr. 2000.

DOI: $10.1054 /$ mehy.1999.0923

PMid: 10859662
7. O. Johansson, "Disturbance of the immune system by electromagnetic fields-A potentially underlying cause for cellular damage and tissue repair reduction which could lead to disease and impairment," Pathophysiology, vol. 16, no. 2-3, pp. 157 - 177, Aug. 2009.

DOI: 10.1016/j.pathophys.2009.03.004

PMid: 19398310

8. C. L. Ross, B.S. Harrison, "An introduction to electromagnetic field therapy and immune function: a brief history and current status," J. of Science and Applications: Biomedicine, vol. 3, no. 2, pp. 18 - 29, 2015 .

Retrieved from:

http://inter-use.com/uploads/soft/150302/1150302163S4.pdf

Retrieved on: Jan. 20, 2017

9. M. Pesce, A. Patruno, L. Speranza, M. Reale, "Extremely Low Frequency Electromagnetic Field and Wound Healing: Implication of Cytokines as Biological Mediators," European Cytokine Network, vol. 24, no. 1, pp. 1 - 10, Mar. 2013.

DOI: $10.1684 /$ ecn.2013.0332

PMid: 23674517

10. F. Guerriero, G. Ricevuti, "Extremely low frequency electromagnetic fields stimulation modulates autoimmunity and immune responses: a possible immuno-modulatory therapeutic effect in neurodegenerative diseases," Neural Regen. Res. vol. 11, no. 12, pp. 1888 - 1895, Dec. 2016.

DOI: $10.4103 / 1673-5374.195277$

PMid: 28197174

PMid: PMC5270416 